\title{
A Phase I, Open-Label, Parallel-Group, Single-Dose Trial of the Pharmacokinetics, Safety, and Tolerability of Cannabidiol in Subjects with Mild to Severe Renal Impairment
}

\author{
Bola Tayo $^{1} \cdot$ Lesley Taylor $^{1} \cdot$ Farhad Sahebkar $^{2} \cdot$ Gilmour Morrison $^{1}$
}

Published online: 5 December 2019

(c) The Author(s) 2019

\begin{abstract}
Introduction As patients who receive cannabidiol (CBD) may have co-existing renal morbidities, it is important to understand whether dose adjustments are necessary to mitigate the risk of exposure-related toxicity. This study was conducted to evaluate the pharmacokinetics, safety, and tolerability of CBD in patients with renal impairment.

Methods The pharmacokinetics and safety of a single oral $200 \mathrm{mg}$ dose of a plant-derived pharmaceutical formulation of highly purified CBD in oral solution (Epidiolex ${ }^{\circledR}$ in the USA; $100 \mathrm{mg} / \mathrm{mL}$ ) were assessed in subjects with mild, moderate, or severe renal impairment ( $n=8$ /group) relative to matched subjects with normal renal function $(n=8)$. Blood samples were collected until $48 \mathrm{~h}$ post-dose and evaluated by liquid chromatography with tandem mass spectrometry. Analysis of variance was used to compare primary pharmacokinetic parameters (maximum measured plasma concentration $\left[C_{\max }\right]$, oral clearance of drug from plasma $[C L / F]$, renal clearance $\left[\mathrm{CL}_{\mathrm{R}}\right]$, area under the plasma concentration-time curve $[\mathrm{AUC}]$ from time zero to last measurable concentration $\left[\mathrm{AUC}_{t}\right]$, and $\mathrm{AUC}$ from time zero to infinity $\left[\mathrm{AUC}_{\infty}\right]$ ); descriptive analysis was used for secondary pharmacokinetic parameters (time to $C_{\max }\left[t_{\max }\right]$, terminal [elimination] half-life $\left[t_{1 / 2}\right]$, cumulative amount excreted from time zero to the last quantifiable sample $\left[\mathrm{Ae}_{\text {last }}\right]$, and fraction of the systemically available drug excreted into the urine $\left.\left[f_{\mathrm{e}}\right]\right)$.

Results No statistically significant differences were observed in $C_{\max }, \mathrm{AUC}_{t}, \mathrm{AUC}_{\infty}$, or $t_{\max }$ values between subjects with mild, moderate, or severe renal impairment and subjects with normal renal function for CBD or its major metabolites, 7-carboxy-CBD (7-COOH-CBD) and 7-hydroxy-CBD (7-OH-CBD), and minor metabolite, 6-hydroxy-CBD (6-OH-CBD); geometric mean ratio for $C_{\max }$ values ranged from 0.68 to 1.35 . No differences were observed for other secondary parameters $\left(\mathrm{Ae}_{\text {last }}\right.$ and $\left.f_{\mathrm{e}}\right)$. CBD, 7-COOH-CBD, 7-OH-CBD, and 6-OH-CBD were highly protein bound (>90\%); binding was similar in all subject groups. Urine analysis for $\mathrm{CBD}$ recorded no appreciable amount, and thus no urinary pharmacokinetic parameters could be derived. Adverse events (AEs) affected two subjects; all five AEs were mild in severity and resolved during the trial. There were no serious AEs or discontinuations due to AEs. Laboratory, physical examination, vital sign, and 12-lead electrocardiogram findings were not clinically significant.
\end{abstract}

Conclusion Renal impairment had no effect on the metabolism of CBD after a single oral $200 \mathrm{mg}$ dose. CBD was generally well tolerated in subjects with varying degrees of renal function.

Registration European Union Clinical Trials Register (EudraCT) no. 2015-002122-39.

Electronic supplementary material The online version of this article (https://doi.org/10.1007/s40262-019-00841-6) contains supplementary material, which is available to authorized users.

Gilmour Morrison

GMorrison@gwpharm.com

1 GW Research Ltd, Sovereign House, Vision Park, Histon, Cambridge CB24 9BZ, UK

2 Greenwich Biosciences, Inc., 5750 Fleet Street, Suite 200, Carlsbad, CA 92008, USA

\section{Introduction}

Highly purified cannabidiol (CBD; Epidiolex $\left.{ }^{\circledR}\right)$ is approved by the Food and Drug Administration (FDA) in the USA for seizures associated with Lennox-Gastaut syndrome (LGS) or Dravet syndrome (DS) in patients $\geq 2$ years of age [1-4]. CBD likely exerts a cumulative anticonvulsant effect via several endogenous systems, including, but not limited to, modulation of intracellular calcium $\left(\mathrm{Ca}^{2+}\right)$ and neuronal 


\section{Key Points}

Following a single oral $200 \mathrm{mg}$ dose of cannabidiol (CBD), renal impairment status was found to have no effect on CBD or its biotransformation products.

No statistically significant differences were observed in maximum measured plasma concentration $\left(C_{\max }\right)$, area under the plasma concentration-time curve (AUC) from time zero to last measurable concentration $\left(\mathrm{AUC}_{t}\right), \mathrm{AUC}$ from time zero to infinity $\left(\mathrm{AUC}_{\infty}\right)$, or time to $C_{\max }\left(t_{\max }\right)$ values between subjects with renal impairment and subjects with normal renal function.

A single dose of $200 \mathrm{mg}$ CBD was well tolerated across all subject groups, and no safety concerns were observed.

excitability [5]. Results from non-clinical studies and the scientific literature support at least three targets underlying the mechanism of anticonvulsant action of CBD: modulation of intracellular $\mathrm{Ca}^{2+}$ by antagonism of $\mathrm{G}$ protein-coupled receptor 55 (GPR55) and desensitization of transient receptor potential vanilloid type 1 (TRPV1) channels, and inhibition of adenosine reuptake via inhibition of the equilibrative nucleoside transporter 1 (ENT1) [5-8]. Importantly, and unlike $\Delta^{9}$-tetrahydrocannabinol (THC), CBD is unlikely to cause euphoric effects associated with propensity for abuse, due to its lack of orthosteric engagement of the cannabinoid receptor type $1\left(\mathrm{CB}_{1}\right)$ at physiologically achievable concentrations [9].

CBD is highly lipophilic and is extensively metabolized by the liver to form monohydroxylated metabolites. The major circulating metabolite in vivo in humans is 7-carboxyCBD (7-COOH-CBD), followed by parent CBD, 7-hydroxyCBD (7-OH-CBD; an active metabolite), and 6-hydroxyCBD (6-OH-CBD; a relatively minor metabolite) [10]. CBD is also subject to a large first-pass effect. Wall et al. [11] found that at $72 \mathrm{~h}$ after dosing, approximately $33 \%$ of CBD and its metabolites were excreted in the feces (of which a large amount was intact) and $16 \%$ were excreted in the urine. It is possible that the patients who receive CBD will have co-existing morbidities, which can lead to drug accumulation. There are no previously published studies investigating the pharmacokinetics of GW Research Ltd's formulation of CBD in renally impaired subjects. This trial aimed to assess the effect of renal impairment on systemic exposure to a single dose of CBD.

\section{Methods}

\subsection{Trial Design}

All relevant trial-related documents were reviewed by independent ethics committees, and approval for the trial was granted on 11 September 2015. All subjects provided written informed consent for participation in the trial, which was performed in full conformity with the current Declaration of Helsinki [12], the International Council for Harmonisation guidelines for Good Clinical Practice [13], and all other applicable regulations. The trial was performed between 14 September 2015 and 10 February 2016 at three Pharmaceutical Research Associates (PRA) sites specializing in clinical pharmacology trials (one each in Hungary, the Czech Republic, and Slovakia). The trial was performed considering the FDA [14] and European Medicines Agency (EMA) [15] recommendations for the evaluation of pharmacokinetics in subjects with impaired renal function.

The trial consisted of a screening period (Days -28 to -2 ), a treatment period (hospitalization from Day -1 until Day 3), and a follow-up visit (Day 14 [ \pm 2 days]). Renal function was assessed by the estimated creatinine clearance (CLcr) using the Cockcroft-Gault equation at screening.

During the in-house treatment period, baseline assessments were performed on Day - 1 (after an overnight fast of at least $8 \mathrm{~h}$ ). On the morning of Day 1, subjects received a standardized low-protein breakfast $2 \mathrm{~h}$ prior to dosing with a single oral $200 \mathrm{mg}$ dose of a pharmaceutical formulation of highly purified CBD derived from Cannabis sativa L. plant in oral solution $\left(100 \mathrm{mg} / \mathrm{mL}\right.$; Epidiolex ${ }^{\circledR}$ in the USA; GW Research Ltd, Cambridge, UK). Results from this study cannot be extrapolated to other CBD-containing products.

Observations were made until release following 48-h post-dose assessments on Day 3. A follow-up visit was performed on Day 14 ( \pm 2 days). Fluid intake except water was prohibited during fasting (from $2 \mathrm{~h}$ pre-dose to $4 \mathrm{~h}$ post-dose).

\subsection{Inclusion and Exclusion Criteria}

\subsubsection{Trial Population}

The inclusion criteria specified that the trial population should consist of male and female subjects (age 18-75 years; body mass index [BMI] $18-35 \mathrm{~kg} / \mathrm{m}^{2}$ ) with mild, moderate, or severe renal impairment, as defined by estimated CLcr, and subjects with normal renal function (matched to renally impaired subjects with respect to age and BMI).

The renal function categories were classified as follows: 
- Group 1: mild renal impairment (CLcr 50-80 mL/min).

- Group 2: moderate renal impairment (CLcr 30 to $<50 \mathrm{~mL} / \mathrm{min}$ ).

- Group 3: severe renal impairment (CLcr $<30 \mathrm{~mL} / \mathrm{min}){ }^{1}$

- Group 4: normal renal function (CLcr $>80 \mathrm{~mL} / \mathrm{min}$ ).

Female subjects were non-pregnant and non-lactating at screening. Male and female subjects agreed to use effective contraception for the duration of the trial and for 3 months and 30 days thereafter, respectively.

Subjects with impaired renal function were included only if deemed to have stable disease status and no history of kidney transplant. In addition, serum albumin concentrations must not have been $<25 \mathrm{~g} / \mathrm{L}$ and hemoglobin concentrations not $<95 \mathrm{~g} / \mathrm{L}(<100 \mathrm{~g} / \mathrm{L}$ in the Czech Republic) at screening and baseline.

\subsection{Trial Assessments}

\subsubsection{Pharmacokinetic Assessments}

At the times specified later in this section, $6 \mathrm{~mL}$ blood samples were taken from subjects via an indwelling intravenous catheter or direct venepuncture into lithium heparin vacutainers; blood samples were then centrifuged for $10 \mathrm{~min}$ at $2600 \mathrm{~g}$ at $18{ }^{\circ} \mathrm{C}$. The resultant plasma was stored upright in a freezer at $-70{ }^{\circ} \mathrm{C}$.

Validated liquid chromatographic-tandem mass spectrometric bioanalytical methods were used to quantify plasma concentrations of CBD, 6-OH-CBD, 7-OH-CBD, and 7-COOH-CBD. For analysis of CBD and metabolites, samples were stored for a maximum of 227 days at $-80^{\circ} \mathrm{C}$, and stability in plasma was determined for at least 309 days for all analytes. As THC is present as a trace impurity $(\leq 0.1 \% \mathrm{w} / \mathrm{w}$ active pharmaceutical ingredient) in the CBD used in this formulation, plasma concentrations of THC and its metabolites 11-hydroxy- $\Delta^{9}$-tetrahydrocannabinol (11-OH-THC) and 11-nor-9-carboxy- $\Delta^{9}$-tetrahydrocannabinol (11-COOHTHC) were also determined.

For analysis of THC and metabolites, samples were stored for a maximum of 244 days at $-80{ }^{\circ} \mathrm{C}$, and stability in plasma was determined for at least 356 days for all analytes.

Blood samples for pharmacokinetic analysis were taken at the following time points:

- Pre-dose, then at $0.25,0.5,0.75,1,1.5,2,2.5,3,4,5,6$, $7,8,9,10,12,24,36$, and $48 \mathrm{~h}$ post-dose.

Urine samples for pharmacokinetic analysis were collected pre-dose and then at intervals of $0-4,4-8,8-12$, 12-24, and 24-48 h post-dose.

\footnotetext{
${ }^{1}$ Subjects requiring dialysis were not enrolled in this trial.
}

Pharmacokinetic parameters were determined by noncompartmental analysis using Phoenix ${ }^{\circledR}$ WinNonlin ${ }^{\circledR}$ (Pharsight Inc., Princeton, NJ, USA) version 6.3. Pharmacokinetic parameters evaluated included maximum measured plasma concentration $\left(C_{\max }\right)$, area under the plasma concentration-time curve (AUC) from time zero to infinity $\left(\mathrm{AUC}_{\infty}\right)$, AUC from time zero to last measurable concentration (AUC t), terminal (elimination) half-life $\left(t_{1 / 2}\right)$, time to $C_{\max }\left(t_{\max }\right)$, cumulative amount excreted from time zero to the last quantifiable sample $\left(\mathrm{Ae}_{\text {last }}\right)$, fraction of the systemically available drug excreted into the urine $\left(f_{\mathrm{e}}\right)$, oral clearance of drug from plasma $(\mathrm{CL} / F)$, renal clearance $\left(\mathrm{CL}_{\mathrm{R}}\right)$, and percentage of estimated extrapolated part for the calculation of $\mathrm{AUC}_{\infty}$ $\left(\left[\mathrm{AUC}_{\infty}-\mathrm{AUC}_{t}\right] / \mathrm{AUC}_{\infty}\right) \times 100\left(\% \mathrm{AUC}_{\mathrm{extra}}\right)$.

\subsubsection{Chromatographic Conditions and Specifications}

High-performance liquid chromatography (HPLC) with tandem mass spectrometry was performed on a Waters Acquity (Waters Corporation, Milford, MA, USA) HPLC system. Chromatographic separations were performed on an Acquity BEH Phenyl Column (internal diameter $1.7 \mu \mathrm{m}$, $2.1 \times 100 \mathrm{~mm}$ ).

The selectivity of the HPLC method was checked by comparing chromatograms from blank plasma with the corresponding spiked peaks. Each blank plasma sample was tested to ensure that there were no significant interfering peaks with any analyte under investigation. The assay ranges were between 2.00 and $10,000 \mathrm{ng} / \mathrm{mL}$ for CBD, 0.250 and $250 \mathrm{ng} /$ $\mathrm{mL}$ for $6-\mathrm{OH}-\mathrm{CBD}, 0.250$ and $1250 \mathrm{ng} / \mathrm{mL}$ for $7-\mathrm{OH}-\mathrm{CBD}$, and 0.250 and $20,000 \mathrm{ng} / \mathrm{mL}$ for $7-\mathrm{COOH}-\mathrm{CBD}$. Calibration standards were between 0.125 and $62.5 \mathrm{ng} / \mathrm{mL}$ for THC and 0.250 and $125 \mathrm{ng} / \mathrm{mL}$ for 11-OH-THC and 11-COOH-THC. All analyte calibration curves were created using weighted least-squares linear/quadratic regression. There were no significant interfering peaks observed at the retention times for any of the analytes, indicating adequate selectivity of the methods.

The precision (coefficient of variation [CV]) and accuracy (relative error $[\mathrm{RE}] /$ mean \% different [Bias]) of the HPLC method were determined by analysis of the plasma samples and were acceptable for all analytes $(\leq 15 \%[20 \%$ at the lower limit of quantification (LLOQ)]). The recovery of CBD, 6-OH-CBD, 7-OH-CBD, and 7-COOH-CBD from human plasma was between 92 and $100 \%$ at three concentrations tested (low, medium, and high) and was considered acceptable. The recovery of THC, 11-OH-THC, and 11-COOH-THC from human plasma was between $56.3 \%$ and $124 \%$ at three concentrations tested (low, medium, and high). Although the recovery for THC and its metabolites varied between quality control (QC) levels, this did not affect the linearity of the assay and therefore was not considered to impact the validity of the data. 
Most analytes passed an assessment of matrix effect in population plasma for renally impaired subjects; however, 11-COOH-THC failed this assessment at the low QC level (suggesting the method did not compensate appropriately for matrix effects). As such, 11-COOH-THC data in subjects with renal impairment should be regarded with a degree of caution.

Protein binding was determined by extracting and analyzing pre-dose plasma that had been spiked with $\mathrm{CBD}, 6-\mathrm{OH}-$ CBD, 7-OH-CBD, 7-COOH-CBD, THC, 11-OH-THC, and 11-COOH-THC and equilibrated for $20-24 \mathrm{~h}$ at $37{ }^{\circ} \mathrm{C}$ to provide a concentration of total analyte content: protein bound + unbound analyte. A supernatant fraction (ultracentrifuged at $45,000 \mathrm{rpm}$ for $20 \mathrm{~h}$ at $37^{\circ} \mathrm{C}$ ) from aliquots of spiked plasma was extracted and analyzed for each subject to provide a concentration of unbound fraction of each analyte.

The bioanalytical methods used in this trial were validated according to guidelines from the EMA [16] and FDA [17]; these methods are further described in the Electronic Supplementary Material.

Various bioanalytical limitations were observed during the protein-binding validation process. During validation of the method for separation of unbound analytes by ultracentrifugation, potential bias due to non-specific binding and analyte instability was observed; as such, total CBD (bound and unbound) and metabolite pharmacokinetic data were used for the primary assessment and are presented in this article.

\subsubsection{Safety Assessments}

The safety and tolerability of CBD were evaluated by recording the incidence of adverse events (AEs) throughout the trial, clinical laboratory tests, vital signs, 12-lead electrocardiography (ECG), and physical examinations.

\subsection{Statistical Analysis}

The primary objective of the trial was to assess the effects of a single dose of CBD on the pharmacokinetic parameters of CBD and its major metabolites in subjects with impaired renal function compared with subjects with normal renal function. Secondary objectives were to assess the safety and tolerability of CBD in the same population. Descriptive statistics of subject demographics and safety outcomes were based on the safety analysis set (all subjects who received CBD).

The pharmacokinetic parameters of CBD, THC, and their metabolites were calculated for the pharmacokinetic analysis set (all subjects who received CBD and had evaluable pharmacokinetic data) using Phoenix ${ }^{\circledR}$ WinNonlin ${ }^{\circledR}$ version 6.3.
Pharmacokinetic parameters for all analytes with sufficient data above LLOQ were estimated from the concentration-time profiles for individuals in the pharmacokinetic analysis set. At least three data points (not including $C_{\max }$ ) were required to calculate $k_{\mathrm{el}}$ (elimination rate constant [from the central compartment]), and percentage extrapolation of $\leq 30 \%$ was required to retain $\mathrm{AUC}_{\infty}$ and $t_{1 / 2}$; subjects who did not satisfy this criterion were excluded from the analysis. Analysis of variance (ANOVA) was used to compare primary pharmacokinetic parameters $\left(C_{\max }, \mathrm{CL} / F, \mathrm{CL}_{\mathrm{R}}\right.$, $\mathrm{AUC}_{\infty}$, and $\mathrm{AUC}_{t}$ ) between the control group of healthy subjects and each of the groups with renal impairment. Pharmacokinetic values were log-transformed prior to analysis. Covariates included sex, age, and BMI, if significant. Geometric least-squares means were used to calculate the ratios of primary pharmacokinetic parameters in each renal impairment group to those in the control group, together with $90 \%$ confidence intervals (CIs). A Wilcoxon rank-sum test with Hodges-Lehmann estimator was used for comparison of the $t_{\text {max }}$ values between the control and each renal impairment group. Estimates of the median differences between groups were determined along with $90 \%$ CIs.

The relationship between log-transformed primary pharmacokinetic parameters and estimated CLcr at screening was explored by a linear regression approach that included sex, age, and BMI, if significant. Secondary pharmacokinetic parameters and safety data were analyzed descriptively.

\subsubsection{Sample Size}

The planned sample size of eight participants per group was based on practical considerations and guidance from the FDA [14] and EMA [15].

\section{Results}

\subsection{Subject Demographics}

A total of 32 subjects were enrolled into one of four subject groups (mild $[n=8]$, moderate $[n=8]$, or severe $[n=8]$ renal impairment or normal renal function $[n=8])$. All 32 subjects completed the trial without any major protocol deviations and were included in the safety and pharmacokinetic analysis sets.

All subjects enrolled were white. The ratio of male to female subjects was 5:3 in the mild and moderate renal impairment groups and 3:5 in the severe renal impairment and normal renal function groups. Mean age across the groups ranged from 58.8 to 64.6 years, and mean BMI ranged from 27.6 to $29.9 \mathrm{~kg} / \mathrm{m}^{2}$ (Table 1 ). 
Table 1 Demographics and baseline characteristics (safety analysis set)

\begin{tabular}{|c|c|c|c|c|}
\hline Characteristics & Mild RI $(n=8)$ & Moderate RI $(n=8)$ & Severe RI $(n=8)$ & Normal RF $(n=8)$ \\
\hline \multicolumn{5}{|l|}{$\operatorname{Sex}[n(\%)]$} \\
\hline Male & $5(62.5)$ & $5(62.5)$ & $3(37.5)$ & $3(37.5)$ \\
\hline Female & $3(37.5)$ & $3(37.5)$ & $5(62.5)$ & $5(62.5)$ \\
\hline \multicolumn{5}{|l|}{$\operatorname{Race}[n(\%)]$} \\
\hline White & $8(100)$ & $8(100)$ & $8(100)$ & $8(100)$ \\
\hline Age (years) [mean (SD)] & $61.9(10.9)$ & $58.8(12.4)$ & $64.6(10.6)$ & $60.4(11.5)$ \\
\hline BMI $\left(\mathrm{kg} / \mathrm{m}^{2}\right)[$ mean (SD)] & $27.6(3.3)$ & $28.5(2.9)$ & $29.9(3.5)$ & $29.6(3.6)$ \\
\hline CLcr (mL/min) [mean (SD)] & $66.9(8.3)$ & $40.0(6.1)$ & $21.7(6.0)$ & $111.7(31.8)$ \\
\hline
\end{tabular}

$B M I$ body mass index, $C L c r$ creatinine clearance, $R F$ renal function, $R I$ renal impairment, $S D$ standard deviation
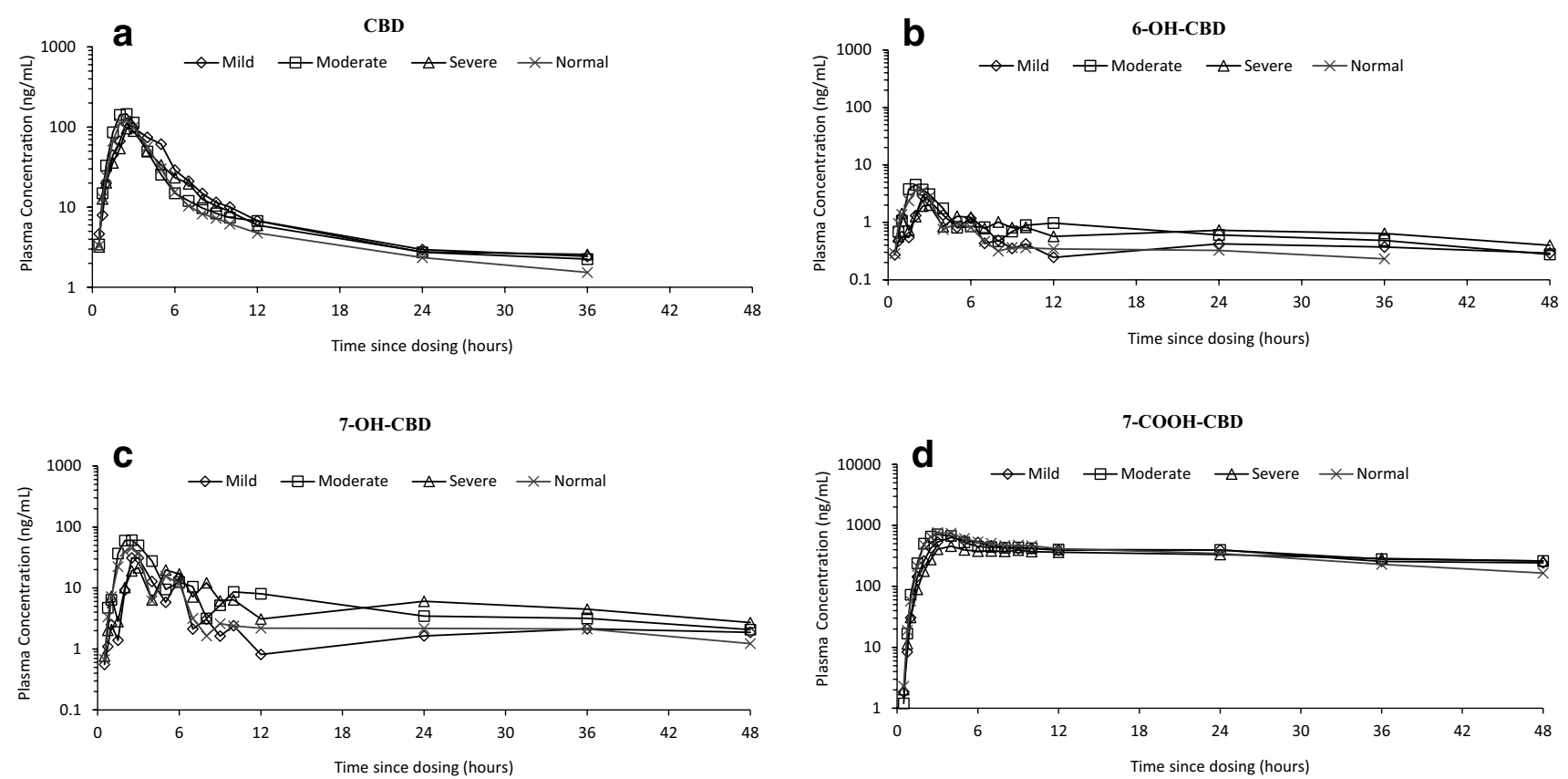

Fig. 1 Geometric mean plasma concentration-time profiles for a cannabidiol (CBD), b 6-hydroxy-cannabidiol (6-OH-CBD), c 7-hydroxycannabidiol (7-OH-CBD), and d 7-carboxy-cannabidiol (7-COOH-

\subsection{Concomitant Medication}

Twenty-six (81.3\%) subjects took at least one concomitant medication during the trial. The most common concomitant medication classes reported were blood pressure-regulating agents ( $\beta$-blockers, xanthine oxidase inhibitors, calcium channel blockers), thyroid hormones, and diuretics. None were considered to impact the safety or interpretation of the trial data.

\subsection{Pharmacokinetics}

Systemic exposure to CBD and its metabolites was unaffected by renal impairment (any severity; Fig. 1).

CBD) after a single oral $200 \mathrm{mg}$ CBD dose, by renal function group (semi-logarithmic) (pharmacokinetics analysis set)

For CBD and its metabolites, there were no significant differences in $C_{\max }$ values between subjects with normal renal function and subjects with renal impairment; geometric mean ratios ranged from 0.68 to 1.35 (Table 2). $\mathrm{AUC}_{t}$ and $\mathrm{AUC}_{\infty}$ (not calculable for 7-COOH-CBD due to a long $t_{1 / 2}$ ) were unaffected by renal impairment when subjects with normal renal function were compared with subjects with renal impairment; geometric mean ratios ranged from 0.95 to 1.74 for $\mathrm{AUC}_{t}$ and from 1.05 to 2.01 for $\mathrm{AUC}_{\infty}$, with no systematic trend to any decrease in $\mathrm{CL} / F$ with increasing severity of renal impairment (Table 2).

Regression analysis showed no apparent relationship between the log-transformed primary pharmacokinetic parameters for $\mathrm{CBD}\left(C_{\max }, \mathrm{AUC}_{t}\right.$, and $\left.\mathrm{AUC}_{\infty}\right)$ and estimated 
Table 2 Comparison of cannabidiol, 6-OH-CBD, 7-OH-CBD, and 7-COOH-CBD pharmacokinetic parameters between renal impairment groups and the normal renal function group (pharmacokinetic analysis set)

\begin{tabular}{|c|c|c|c|c|c|}
\hline Comparison & $\begin{array}{l}C_{\max }[\text { geometric } \mathrm{LS} \\
\text { mean ratio }(90 \% \mathrm{CI})]\end{array}$ & $\begin{array}{l}\mathrm{AUC}_{\infty} \text { [geometric } \mathrm{LS} \\
\text { mean ratio }(90 \% \mathrm{CI})]\end{array}$ & $\begin{array}{l}\mathrm{AUC}_{t}[\text { geometric LS } \\
\text { mean ratio }(90 \% \mathrm{CI})]\end{array}$ & $\begin{array}{l}\mathrm{CL} / F \text { [geometric LS } \\
\text { mean ratio }(90 \% \mathrm{CI})]\end{array}$ & $t_{\max }[\text { difference }(90 \% \mathrm{CI})]^{\mathrm{a}}(\mathrm{h})$ \\
\hline \multicolumn{6}{|l|}{ CBD } \\
\hline Mild/normal & $1.31(0.73-2.35)$ & $1.20(0.59-2.45)$ & $1.44(0.83-2.51)$ & $0.83(0.41-1.69)$ & $0.25(0.00-1.00)$ \\
\hline Moderate/normal & $1.12(0.62-2.02)$ & $1.05(0.56-1.96)$ & $1.14(0.66-1.98)$ & $0.96(0.51-1.79)$ & $0.00(-0.50$ to 0.00$)$ \\
\hline Severe/normal & $1.02(0.57-1.83)$ & $1.20(0.64-2.25)$ & $1.15(0.66-1.99)$ & $0.83(0.44-1.56)$ & $0.00(0.00-0.50)$ \\
\hline \multicolumn{6}{|l|}{ 6-OH-CBD } \\
\hline Mild/normal & $0.94(0.66-1.35)$ & $1.58(0.98-2.55)$ & $1.38(0.83-2.31)$ & & $0.25(-0.75$ to 1.50$)$ \\
\hline Moderate/normal & $1.14(0.80-1.63)$ & $2.01(1.25-3.25)$ & $1.49(0.90-2.49)$ & & $-0.50(-0.50$ to 0.00$)$ \\
\hline Severe/normal & $1.01(0.71-1.43)$ & $1.65(1.07-2.54)$ & $1.74(1.04-2.90)$ & & $0.38(-0.50$ to 1.00$)$ \\
\hline \multicolumn{6}{|l|}{ 7-OH-CBD } \\
\hline Mild/normal & $0.85(0.54-1.36)$ & $1.14(0.81-1.59)$ & $1.08(0.77-1.53)$ & & $0.25(-0.50$ to 1.50$)$ \\
\hline Moderate/normal & $1.35(0.85-2.15)$ & $1.37(0.98-1.91)$ & $1.32(0.94-1.86)$ & & $0.00(-0.50$ to 0.50$)$ \\
\hline Severe/normal & $1.10(0.69-1.75)$ & $1.56(1.12-2.19)$ & $1.42(1.01-2.00)$ & & $0.00(-0.50$ to 0.50$)$ \\
\hline \multicolumn{6}{|l|}{ 7-COOH-CBD } \\
\hline Mild/normal & $0.88(0.50-1.54)$ & & $1.04(0.69-1.55)$ & & $0.00(-0.50$ to 1.00$)$ \\
\hline Moderate/normal & $0.89(0.50-1.56)$ & & $1.07(0.72-1.60)$ & & $0.00(-0.50$ to 0.50$)$ \\
\hline Severe/normal & $0.68(0.39-1.19)$ & & $0.95(0.63-1.41)$ & & $1.00(0.00-1.50)$ \\
\hline
\end{tabular}

6-OH-CBD 6-hydroxy-cannabidiol, 7-COOH-CBD 7-carboxy-cannabidiol, 7- $O H$ - $C B D$ 7-hydroxy-cannabidiol, $A U C_{\infty}$ area under the plasma concentration-time curve from time zero to infinity, $A U C_{t}$ area under the plasma concentration-time curve from time zero to last measurable concentration, $C B D$ cannabidiol, $C I$ confidence interval, $C L / F$ oral clearance of drug from plasma, $C_{\max }$ maximum measured plasma concentration, $L S$ least-squares, $t_{\max }$ time to $C_{\max }$

${ }^{\text {a } H o d g e s-L e h m a n n ~ e s t i m a t e ~}(90 \% \mathrm{CI})$

CLcr (at screening) in all subject groups ( $p$ values $>0.05$ [range 0.1-1]).

The $t_{\max }$ values for CBD and the metabolites 6-OH-CBD and 7-OH-CBD were independent of renal impairment status, with geometric mean plasma $t_{\max }$ reached between 2 and $3 \mathrm{~h}$ post-dose for all subject groups. The $t_{\max }$ for $7-\mathrm{COOH}-$ CBD appeared slightly later but was also independent of renal impairment status, with geometric mean plasma $t_{\max }$ reached between approximately 3 and $4 \mathrm{~h}$ (range $2-9 \mathrm{~h}$ ) postdose for all subject groups. Statistical analysis showed that renal impairment had no effect on $t_{\max }$ values for CBD or any of its metabolites (Table 2). 7-COOH-CBD was the most abundant circulating product in plasma, followed by CBD, 7-OH-CBD, and then 6-OH-CBD (Fig. 1).

The $t_{1 / 2}$ values for CBD, 6-OH-CBD, and 7-OH-CBD ranged from 11 to $22 \mathrm{~h}$ in all subject groups. Although statistical analyses were not performed for $t_{1 / 2}$ values, there were no clear trends between subjects with normal renal function and subjects with renal impairment (Table 3). For 7-COOH$\mathrm{CBD}, t_{1 / 2}$ was longer than the 48 - $\mathrm{h}$ sampling time for all renal function groups. As such, $t_{1 / 2}$ values for 7-COOH-CBD were not calculated (Table 3 ).

For CBD, CL/F ranged from 351 to $510 \mathrm{~L} / \mathrm{h}$ and the apparent volume of distribution $\left(\mathrm{V}_{\mathrm{z}} / F\right)$ ranged from 5800 to 7778 L. No statistically significant differences in CL/ $F$ were observed between renal impairment groups (mild to severe) and subjects with normal renal function; geometric mean ratios ranged from 0.83 to 0.96 and all $90 \%$ CIs of ratios contained 1 (Table 2).

Urine analysis for CBD recorded no appreciable amount of CBD. Concentrations were below the LLOQ $(<0.125 \mathrm{ng} /$ $\mathrm{mL}$ ) for most subjects at most time points and thus the primary parameter $\mathrm{CL}_{\mathrm{R}}$ and the secondary parameters $\mathrm{Ae}_{\text {last }}$ and $f_{\mathrm{e}}$ were not reported.

THC and its major metabolites were either not detected or detected only in trace concentrations $(\leq 1.93 \mathrm{ng} / \mathrm{mL})$ in plasma and were independent of renal impairment status.

\subsection{Plasma Protein Binding of Cannabidiol (CBD)}

There was no trend observed between the degree of renal impairment and plasma protein binding. CBD and its major metabolites were all highly bound to plasma proteins. The extent of protein binding ranged from 86.7 to $92.2 \%$ bound for CBD and from 96.8 to $99.0 \%$ bound for its major metabolites. Data could only be considered qualitative due to bioanalytical issues (the process and stability of the free fraction was not supported during bioanalytical method validation). The results from the free unbound data were consistent with the plasma data; however, only total drug (bound and unbound) pharmacokinetic parameters are presented in this article, as they can be supported by validation. 
Table 3 Pharmacokinetic parameters for cannabidiol, 6-OH-CBD, 7-OH-CBD, and 7-COOH-CBD (pharmacokinetic analysis set)

\begin{tabular}{|c|c|c|c|c|c|c|c|c|}
\hline $\begin{array}{l}\text { Renal function } \\
\text { group }\end{array}$ & $\begin{array}{l}C_{\max }(\mathrm{ng} / \mathrm{mL})^{\mathrm{a}} \\
(n=8)\end{array}$ & $\begin{array}{l}t_{\max } \\
(\mathrm{h})^{\mathrm{b}} \\
(n=8) \\
\end{array}$ & $\begin{array}{l}t_{1 / 2} \\
(\mathrm{~h})^{\mathrm{c}, \mathrm{d}} \\
(n=8)\end{array}$ & $\begin{array}{l}\mathrm{AUC}_{t}(\mathrm{ng} \cdot \mathrm{h} / \\
\mathrm{mL})^{\mathrm{a}} \\
(n=8)\end{array}$ & $\begin{array}{l}\mathrm{AUC}_{\infty}(\mathrm{ng} \cdot \mathrm{h} / \\
\mathrm{mL})^{\mathrm{a}, \mathrm{d}} \\
(n=8)\end{array}$ & $\begin{array}{l}\% \mathrm{AUC}_{\mathrm{extra}}^{\mathrm{a}, \mathrm{e}} \\
(n=8)\end{array}$ & $\begin{array}{l}\mathrm{CL} / F(\mathrm{~L} / \mathrm{h})^{\mathrm{c}} \\
(n=8)\end{array}$ & $\begin{array}{l}\mathrm{V}_{\mathrm{z}} / F(\mathrm{~L})^{\mathrm{c}} \\
(n=8)\end{array}$ \\
\hline \multicolumn{9}{|l|}{ CBD } \\
\hline Mild RI & $200(42.7)$ & $2.5(1.5-5.0)$ & $15.5^{f}(64.5)$ & $671(40.9)$ & $600^{\mathrm{g}}(50.0)$ & $7.50^{\mathrm{g}}(73.0)$ & $365^{\mathrm{g}}(52.3)$ & $6661^{\mathrm{g}}(55.5)$ \\
\hline Moderate RI & $172(85.3)$ & $2.0(2.0-3.0)$ & $14.6^{\mathrm{f}}(46.6)$ & $530(74.4)$ & $522^{\mathrm{f}}(63.6)$ & $7.99^{f}(55.5)$ & $434^{\mathrm{f}}(50.4)$ & $7778^{f}(58.0)$ \\
\hline Severe RI & $155(40.6)$ & $2.5(1.5-7.0)$ & $13.1^{\mathrm{f}}(41.5)$ & $532(32.7)$ & $601^{\mathrm{f}}(35.9)$ & $8.63^{f}(31.3)$ & $351^{\mathrm{f}}(37.3)$ & $6016^{\mathrm{f}}(39.9)$ \\
\hline Normal RF & $153(74.7)$ & $2.5(2.0-3.0)$ & $11.2(47.2)$ & 464 (77.6) & 499 (76.6) & $6.81(27.4)$ & $510(87.6)$ & $5800(29.2)$ \\
\hline \multicolumn{9}{|l|}{ 6-OH-CBD } \\
\hline Mild RI & $4.12^{\mathrm{f}}(59.0)$ & $2.5^{\mathrm{f}}(0.8-5.0)$ & $19.3^{\mathrm{h}}(6.6)$ & $30.5^{\mathrm{f}}(63.1)$ & $61.2^{\mathrm{h}}(3.3)$ & $16.3^{\mathrm{h}}(25.4)$ & & \\
\hline Moderate RI & $5.17^{f}(32.1)$ & $2.0^{\mathrm{f}}(1.5-2.5)$ & $22.0^{\mathrm{h}}(38.1)$ & $35.5^{\mathrm{f}}(78.5)$ & $74.9^{\mathrm{h}}(2.1)$ & $19.5^{\mathrm{h}}(47.5)$ & & \\
\hline Severe RI & $3.98^{f}(31.8)$ & $2.5^{\mathrm{f}}(1.0-6.0)$ & $20.2^{\mathrm{g}}(30.8)$ & $39.2^{\mathrm{f}}(48.8)$ & $55.9^{\mathrm{g}}(52.6)$ & $21.1^{\mathrm{g}}(35.8)$ & & \\
\hline Normal RF & $3.86^{\mathrm{i}}(18.2)$ & $2.5^{\mathrm{i}}(2.0-3.0)$ & $15.0^{\mathrm{h}}(45.4)$ & $22.6^{\mathrm{i}}(51.7)$ & $34.3^{\mathrm{h}}(54.8)$ & $17.2^{\mathrm{h}}(14.9)$ & & \\
\hline \multicolumn{9}{|l|}{ 7-OH-CBD } \\
\hline Mild RI & $44.6(77.0)$ & $2.8(1.0-5.0)$ & $19.8(15.9)$ & $327.3(60.2)$ & 381.7 (59.6) & $13.8(25.0)$ & & \\
\hline Moderate RI & $70.5(30.0)$ & $2.3(2.0-3.0)$ & $18.1(26.3)$ & $400.0(23.7)$ & $457.6(25.0)$ & $11.4(50.0)$ & & \\
\hline Severe RI & $61.9^{j}(37.6)$ & $3.0^{\mathrm{j}}(2.0-6.0)$ & $19.1^{\mathrm{j}}(13.7)$ & $448.1^{\mathrm{j}}(15.5)$ & $520.8^{\mathrm{j}}(17.0)$ & $13.8^{\mathrm{j}}(13.7)$ & & \\
\hline Normal RF & $52.3(34.8)$ & $2.5(2.0-4.0)$ & $16.6(30.6)$ & $302.3(22.6)$ & $335.1(20.7)$ & $8.4(56.0)$ & & \\
\hline \multicolumn{9}{|l|}{ 7-COOH-CBD } \\
\hline Mild RI & $648.3^{\mathrm{i}}(109.5)$ & $4.0^{\mathrm{i}}(2.5-6.0)$ & $\mathrm{NC}$ & $\begin{array}{c}15,676.7^{\mathrm{i}} \\
(69.2)\end{array}$ & $\mathrm{NC}$ & $\mathrm{NC}$ & & \\
\hline Moderate RI & $578.4^{\mathrm{g}}(32.9)$ & $2.5^{\mathrm{g}}(2.5-4.0)$ & $\mathrm{NC}$ & $\begin{array}{c}14,629.0^{\mathrm{g}} \\
(29.2)\end{array}$ & $\mathrm{NC}$ & $\mathrm{NC}$ & & \\
\hline Severe RI & $571.9(51.2)$ & $4.0(2.5-9.0)$ & $\mathrm{NC}$ & $15,420.1(37.5)$ & $\mathrm{NC}$ & $\mathrm{NC}$ & & \\
\hline Normal RF & $842.5^{\mathrm{g}}(49.8)$ & $3.0(2.5-5.0)$ & $\mathrm{NC}$ & $16,301.6(33.1)$ & $\mathrm{NC}$ & $\mathrm{NC}$ & & \\
\hline
\end{tabular}

6-OH-CBD 6-hydroxy-cannabidiol, 7- $\mathrm{COOH}-\mathrm{CBD}$ 7-carboxy-cannabidiol, 7- $\mathrm{OH}-\mathrm{CBD}$ 7-hydroxy-cannabidiol, $A U \mathrm{C}_{\infty}$ area under the plasma concentration-time curve from time zero to infinity, $A U C_{t}$ area under the plasma concentration-time curve from time zero to last measurable concentration, $C B D$ cannabidiol, $C L / F$ oral clearance of drug from plasma, $C_{\max }$ maximum measured plasma concentration, $C V$ coefficient of variation, $N C$ not calculable, $R F$ renal function, $R I$ renal impairment, $t_{1 / 2}$ terminal (elimination) half-life, $t_{\max }$ time to $C_{\max }, V_{t} / F$ apparent volume of distribution

${ }^{\mathrm{a}}$ Geometric mean (geometric CV)

${ }^{\mathrm{b}}$ Median (range)

${ }^{\mathrm{c}}$ Arithmetic mean $(\mathrm{CV})$

${ }^{\mathrm{d}}$ Percentage extrapolation $\leq 30 \%$ was required to retain $\mathrm{AUC}_{\infty}$ and $t_{1 / 2}$; subjects who did not satisfy this criterion were excluded from the analysis ${ }^{\mathrm{e}} \% \mathrm{AUC}_{\mathrm{extra}}$ is the percentage of estimated extrapolated part for the calculation of $\mathrm{AUC}_{\infty}\left(\left[\mathrm{AUC}_{\infty}-\mathrm{AUC}_{t}\right] / \mathrm{AUC} \mathrm{C}_{\infty}\right) \times 100$

${ }^{\mathrm{f}} n=6$

$\mathrm{g}_{n=4}$

$\mathrm{h}_{n=2}$

${ }^{\mathrm{i}} n=5$

${ }^{\mathrm{j}} n=7$

\subsection{Safety}

A single oral $200 \mathrm{mg}$ dose of CBD was well tolerated in all subject groups; all AEs were mild in severity and resolved during the trial. There were no serious AEs, deaths, AEs of special interest, pregnancies, or early withdrawals due to AEs.

There were only five AEs reported throughout the trial, and no AEs were reported in the moderate or severe renal impairment groups. One subject in the mild renal impairment group reported three AEs: one case each of visual disturbance, nausea, and drowsiness. One subject in the normal renal function group reported two AEs: one case each of back pain and pain in hip.

There were no clinically significant changes for any laboratory parameter, and no laboratory abnormalities were considered AEs. There were no clinically significant physical examination, vital sign, or ECG findings. 


\section{Discussion}

This trial is the first to investigate the pharmacokinetics of this oral formulation of CBD in subjects with renal impairment. As patients who receive CBD may have co-existing renal morbidities, it is important to understand whether dose adjustments are necessary to mitigate the risk of exposurerelated toxicity $[14,15]$.

\subsection{CBD and Metabolite Pharmacokinetics}

Following a single oral $200 \mathrm{mg}$ dose of CBD, renal impairment status was found to have no effect on CBD or its biotransformation products. No statistically significant differences were observed in $C_{\max }, \mathrm{AUC}_{t}, \mathrm{AUC}_{\infty}$, or $t_{\max }$ values between subjects with renal impairment and subjects with normal renal function. $\mathrm{CBD}$ and its major metabolites were not detected (below LLOQ of assay) in urine, and thus this likely represents a minor route of elimination of intact drug. No differences were observed for any other secondary parameters.

Exposure to 7-COOH-CBD was much greater than that to the parent drug; however, in contrast to $\mathrm{CBD}$, exposure to 7-COOH-CBD was lowest in subjects with severe renal impairment (compared with the other impairment groups and the normal renal function group). A possible explanation for this is the potential for a different biotransformation pathway for 7-COOH-CBD in severely renally impaired subjects [18].

\subsection{Safety}

A single dose of $200 \mathrm{mg}$ CBD was well tolerated across all subject groups, and no safety concerns were observed. Only five mild AEs were reported by two subjects during the trial: one in the mild renal impairment group and one in the normal renal function group. There was no increase in $\mathrm{AE}$ frequency or severity with increasing degree of renal impairment. There were no moderate or severe AEs, deaths, serious AEs, or any other significant events. There were no clinically significant safety findings for laboratory parameters, physical examinations, vital signs, ECG, or body weight.

\subsection{Trial Limitations}

This trial was conducted prior to the availability of data from the multiple-dose pharmacokinetic trials that defined the $t_{1 / 2}$ for CBD. Subsequent trials, now that pharmacokinetic data are available, have been designed to evaluate pharmacokinetic effects over longer time frames. Although data are limited to $48 \mathrm{~h}$ post-dose, the pharmacokinetic results during the first $48 \mathrm{~h}$ are consistent with pharmacokinetic results reported in other single-dose studies with CBD [10].

For 7-COOH-CBD, the $t_{1 / 2}$ was longer than the 48 -h sampling time for all renal function groups. As such, $t_{1 / 2}$ values for 7-COOH-CBD were not calculated. However, $C_{\max }$ and $\mathrm{AUC}_{\text {last }}$ were the primary parameters for the evaluation of renal insufficiency and had no impact on the study results or conclusions. In addition, exposure data from plasma total drug (bound and unbound) pharmacokinetic parameters are presented here, as they can be supported by validation. It should be noted that although data generated for the unbound (free) drug during this study were only considered qualitative, the results were consistent with the total plasma data.

Results from this trial suggest that intact CBD parent drug is not significantly cleared by renal elimination; however, this trial was not designed to evaluate conjugated metabolites in the urine and therefore does not rule out the possibility that CBD metabolites are cleared by conjugation. CBD has not been tested in patients with end-stage renal disease, and it is not known if CBD and its metabolites are dialyzable.

Validation studies have been conducted for potential interference with concomitant medications commonly used to treat the approved indications for CBD; however, because this trial evaluated a special population, renal impairment, it was not possible to conduct validation studies for all possible concomitant medications within this rare subgroup.

\section{Conclusion}

Renal impairment status had no effect on CBD pharmacokinetics following a single oral $200 \mathrm{mg}$ dose, with no statistically significant effects on $C_{\max }, \mathrm{AUC}_{t}, \mathrm{AUC}_{\infty}$, or $t_{\max }$. $\mathrm{CBD}$ was generally well tolerated; there were no serious or severe AEs, and no new safety concerns were identified.

Acknowledgements The authors would like to thank the volunteers who took part in the trial, as well as the staff that assisted with the trial at each site. The authors would also like to acknowledge and thank Nateisha Williams, BS, employee of Greenwich Biosciences, Inc., for providing medical writing support, and Laura Riordan, MS, for providing editorial support funded by Greenwich Biosciences, Inc.

\section{Compliance with Ethical Standards}

Funding This trial was sponsored by GW Research Ltd.

Conflict of interest Bola Tayo, MD, and Gilmour Morrison, HND, are employees of GW Research Ltd and own shares in GW Pharmaceuticals Plc. Lesley Taylor, PhD, was employed at GW Research Ltd at the time this work was completed. Farhad Sahebkar, MD, is an employee of Greenwich Biosciences, Inc. 
Data Availability Statement The sponsor is adhering to current US and EU requirements so will not make individual de-identified participant data available; however, the protocol and statistical analysis plan will be made available upon request to the corresponding author.

Ethical Standards All procedures performed in studies involving human participants were in accordance with the institutional and/or national research committee and with the 1964 Helsinki Declaration and its later amendments or comparable ethical standards. For information on local ethics committees involved in conducting the trial, refer to EudraCT no. 2015-002122-39.

Informed Consent Informed consent was obtained from all individual participants included in the studies.

Open Access This article is distributed under the terms of the Creative Commons Attribution-NonCommercial 4.0 International License (http://creativecommons.org/licenses/by-nc/4.0/), which permits any noncommercial use, distribution, and reproduction in any medium, provided you give appropriate credit to the original author(s) and the source, provide a link to the Creative Commons license, and indicate if changes were made.

\section{References}

1. Devinsky O, Cross JH, Laux L, Cannabidiol in Dravet Syndrome Study Group, et al. Trial of cannabidiol for drug-resistant seizures in the Dravet syndrome. N Engl J Med. 2017;376(21):2011-20.

2. Devinsky O, Patel AD, Thiele EA, et al. Randomized, dose-ranging safety trial of cannabidiol in Dravet syndrome. Neurology. 2018;90(14):e1204-11.

3. Devinsky O, Patel AD, Cross JH, et al. Effect of cannabidiol on drop seizures in the Lennox-Gastaut syndrome. N Engl J Med. 2018;378(20):1888-97.

4. Thiele EA, Marsh ED, French JA, et al. Cannabidiol in patients with seizures associated with Lennox-Gastaut syndrome (GWPCARE4): a randomised, double-blind, placebo-controlled phase 3 trial. Lancet. 2018;391(10125):1085-96.

5. Ibeas Bih C, Chen T, Nunn AV, Bazelot M, Dallas M, Whalley BJ. Molecular targets of cannabidiol in neurological disorders. Neurotherapeutics. 2015;12(4):699-730.

6. Iannotti FA, Hill CL, Leo A, et al. Nonpsychotropic plant cannabinoids, cannabidivarin (CBDV) and cannabidiol (CBD), activate and desensitize transient receptor potential vanilloid 1 (TRPV1) channels in vitro: potential for the treatment of neuronal hyperexcitability. ACS Chem Neurosci. 2014;5(11):1131-41.

7. Yang H, Zhou J, Lehmann C. GPR55-a putative "type 3" cannabinoid receptor in inflammation. J Basic Clin Physiol Pharmacol. 2016;27(3):297-302.
8. Carrier EJ, Auchampach JA, Hillard CJ. Inhibition of an equilibrative nucleoside transporter by cannabidiol: a mechanism of cannabinoid immunosuppression. Proc Natl Acad Sci USA. 2006;103(20):7895-900.

9. Schoedel KA, Szeto I, Setnik B, et al. Abuse potential assessment of cannabidiol (CBD) in recreational polydrug users: a randomized, double-blind, controlled trial. Epilepsy Behav. 2018;88:162-71.

10. Taylor L, Gidal B, Blakey G, Tayo B, Morrison G. A phase I, randomized, double-blind, placebo-controlled, single ascending dose, multiple dose, and food effect trial of the safety, tolerability and pharmacokinetics of highly purified cannabidiol in healthy subjects. CNS Drugs. 2018;32(11):1053-67.

11. Wall ME, Brine DR, Perez-Reyes M. Metabolism of cannabinoids in man. In: Braude MC, Szara S, editors. The pharmacology of marihuana. New York: Raven Press; 1976. p. 93-113.

12. World Medical Association Declaration of Helsinki. Ethical principles for medical research involving human subjects. Edinburgh: World Medical Association; 2000 Oct, including the footnote of October 2002

13. ICH harmonised tripartite guideline: guideline for good clinical practice E6(R1). Geneva: International Council for Harmonisation of Technical Requirements for Pharmaceuticals for Human Use; 1996 Jun.

14. US Food and Drug Administration (FDA). Guidance for industry: pharmacokinetics in patients with impaired renal function-study design, data analysis, and impact on dosing and labeling. 1998. http://www.fda.gov/downloads/Drugs/GuidanceComplianceRe gulatoryInformation/Guidances/ucm072127.pdf. Accessed 31 Aug 2018.

15. Committee for Medicinal Products for Human Use (CHMP) Note for guidance on the evaluation of the pharmacokinetics of medicinal products in patients with impaired renal function. 2004. http://www.ema.europa.eu/docs/en_GB/document_library/Scien tific_guideline/2009/09/WC500003123.pdf. Accessed 31 Aug 2018.

16. European Medicines Agency. Guideline on bioanalytical method validation. $2011 \mathrm{Jul} 21$. https://www.ema.europa.eu/en/docum ents/scientific-guideline/guideline-bioanalytical-method-valid ation_en.pdf. Accessed 30 Aug 2019.

17. US Department of Health and Human Services, Food and Drug Administration (FDA), Center for Drug Evaluation and Research (CDER), Center for Veterinary Medicine (CVM). Guidance for industry, bioanalytical method validation. Rockville: US Department of Health and Human Services, FDA, CDER; 2001.

18. Tarantino G, Di Minno MN, Capone D. Drug-induced liver injury: is it somehow foreseeable? World J Gastroenterol. 2009;15(23):2817-33. 